\title{
Genotype of recipient mother modulate body composition and immunocompetence of transferred progenies
}

\author{
L. Gerlinskaya ${ }^{1 *}$, E. Litvinova ${ }^{1}$, G. Kontsevaya ${ }^{1}$, N. Feofanova ${ }^{1,2}$, K. Achasova ${ }^{1}$, \\ M. Anisimova ${ }^{1}, \mathrm{~S}$. Maslennikova ${ }^{1}, \mathrm{M}$. Zolotykh ${ }^{1}, \mathrm{M}$. Moshkin ${ }^{1}$ \\ ${ }^{1}$ Institute of Cytology and Genetics SB RAS, Novosibirsk, Russia \\ ${ }^{2}$ Institute of Immunology \\ *e-mail: lgerlinskaya@gmail.com
}

Key words: embryos, mothers, immunogenetic dialogue, modulations, offspring properties

Motivation and Aim: Among the causes of persistent phenotypic modifications, the genotype of the nurturing mother, which as a rule differs from the genotype of the transplanted embryo. During fetal development between the mother and the embryo there is a bilateral exchange of immunologically significant information the nature of which is determined by the degree of antigenic differences between mother and embryo, in particularly between genes of the main histocompatibility complex (MHC) [1]. In experiments with $\mathrm{C} 57 \mathrm{BL}$ mice $\left(\mathrm{H} 2^{\mathrm{b}}\right.$ haplotype) and BALB / c ( $\mathrm{H} 2^{\mathrm{d}}$ haplotype) were previously showed, that offspring obtained by interlinear embryo transfers are different from those obtained by intrastrain embryo transplantation by a less adrenocortical response to various variants of social stress [2]. However, this study does not cover the whole variety of immunogenic interactions between the fetus and the mother and the whole variety of potential modulations of the offspring properties caused by the conditions of intrauterine development that can substantially vary depending on the genotype of the nurturing female.

Methods and Algorithms: In the presented study, transplantation of two-cell inbred strain embryos coinciding or different in the MHC (H2) haplotype from the recipient female was used to model three variants of the immunogenic dialogue in the mother-fetus system. 1. A bidirectional immunogenic dialogue - embryos C57BL/6J (H2 ${ }^{\mathrm{b}}$ haplotype) and nurturing BALB/c mothers $\left(\mathrm{H} 2^{\mathrm{d}}\right.$ haplotype). 2. One-way dialogue - embryos $\mathrm{C} 57 \mathrm{BL} / 6 \mathrm{~J}$ and not capable to effectively respond to foreign antigens and immunodeficient NOD.SCID mothers (H2 ${ }^{\mathrm{g} 7}$ haplotype). 3. Absence of immunogenetic dialogue - embryos and mothers of the $\mathrm{C} 57 \mathrm{BL} / 6 \mathrm{~J}$ mice of the same $\mathrm{H} 2^{\mathrm{b}}$ haplotype. In the offspring, at the time of weaning from mothers and at reaching the sexually mature age, the body weight, its composition, as well as the parameters of nonspecific and specific immune responses to the action of Anthrax and Helicobacter hepaticus were measured

Results: Adult descendants born to mothers of the same genetic strain were characterized by a high fat content compared to that of offspring that were born to females of a different genotype. C57BL/6J progenies nurtured by immunodeficient NOD SCID mothers had a more pronounced humoral immune response to oral infection with $H$. hepaticus and less in vitro macrophage cytokine reaction on the anthrax exotoxin in comparison with the offspring nurtured by the C57BL and $\mathrm{BALB} / \mathrm{c}$ mothers.

Conclusion: These results let us to conclude that the influence of the genotype dependent motherfetus dialogue on the fat metabolism and the immune system in adult offspring.

Acknowledgements: Supported by the FPI (6/099/2017ав). The studies are implemented using the equipment of the Center for Genetic Resources of Laboratory Animals at ICG SB RAS, supported by the Ministry of Education and Science of Russia (Unique identifier of the project RFMEFI62117X0015).

References

1. Gerlinskaya L.A., Evsikov V.I. (2001) Influence of genetic dissimilarity of mother and fetus on progesterone concentrations in pregnant mice and adaptive features of offspring. Reproduction. 121(3): 409-17.

2. Bracamonte-Baran W., Burlingham W. (2015) Non-inherited maternal antigens, pregnancy, and allotolerance. Biomed J. 38(1): 39-51. 